\section{ambient \\ SCIENCE \\ Vol. 04(Sp1):16-18 \\ Year 2017}

\title{
The Efficacy of Cognitive-behavioural and Mental Health of Cancer Patients: an Impact of Workshop on the Residents Radiation- Oncology in a Small Group
}

\section{Maryam Javaherian', Parastoo Hajian, Hamidreza Mirzaei ${ }^{2 *}$}

'Young Researchers and Elite Club, Central Tehran Branch, Islamic Azad University, Tehran, Iran.

${ }^{2}$ Cancer Research Center, Shohadae Tajrish Hospital, Department of Radiation Oncology, Shahid Beheshti University of Medical Sciences, Tehran, Iran

Study Area: Tehran, Iran

Coordinates: $35^{\circ} 41^{\prime} 46^{\prime \prime} \mathrm{N} 51^{\circ} 25^{\prime} 23^{\prime \prime} \mathrm{E}$

Key words: Education, Cognitive behavioral therapy Patients with mental health, Residents

\section{Introduction:}

After diagnosis and while treatment, cancer patients undergo a sort of mental depression due to sadness; anxiety, shame, loneliness and frustration which ultimately lead to the poor agreement with the conditions. Such peoples while treatment gets some changes in physical appearance thus they often feel uncomfortable in interpersonal situations, social anxiety, and anger toward family members (spouse and children; Soroush et al., 2015). Identifying the behavioural reactions and thoughts of patients by the medical staffs could be effective in communication between patient and physician, in addition, help patient while treatment and follow-up process. The rapid advance of medical science, changes in health care delivery, patient expectations, places of teaching and learning, the emergence of new areas of science, such as psycho-oncology; the extent of the medical community and patients thus the medical community education becomes necessary (Omrani et al., 2013). In nowadays, the study of the relationship between physician and patient in health care has attracted much attention. In the last two decades, descriptive and experimental research in the field of advance medical advice related to the communication process has increased. However, the results of such efforts are still

\section{Abstract}

While diagnosed, during treatment and even after recovery from the disease, the cancer patient faces various tensions that could reflect in their beliefs and behaviours which could also affect the further treatment processes. On the other hand, effective communication between patient and doctor even patients' files identify errors by their therapists, can increase the confidence level of the patients which may reduce stress while treatment. The aim of this study was to verify the effectiveness of cognitive-behavioural dependent the mental health of cancer patients, the residents of radiotherapy - oncology in a small group. The study was the quasi-experimental type where the data gathering tool was a questionnaire consisted of 12 questions to examine the cognitive errors. Results suggest that implementing the educational programs, medical assistants can effectively identify patients by therapists in their cognitive errors and improving patient-physician relationship.

limited. One of the main reasons for such complex relationship between doctor and patient is compared to other interpersonal communication. Since the relationship between two persons who are not of the same terms and conditions, are of vital importance and therefore quite emotional communication requires a close two-way communication. Some studies have shown that patient, their medical knowledge and capability could be judged by his technical behaviour. Other studies show that the basis of how doctors assess patients, the quality of interpersonal skills (Spencer, 2003). Recently, researchers in communication have a special attention to social issues psychology cancer (Lowe et al., 2005). Most cancer patients are actively seeking information. While $63 \%$ of them feel that doctors should decision-making process, have the primary responsibility. Only $10 \%$ of their considered for a major role in decision-making. A recent study showed that women whose breast cancer is newly diagnosed, doctors prefer the control and treatment of such patient with this therapy, will also assume responsibility for the results of treatment. If the disease recurs, patients would feel that they were taken a wrong decision. In other words, what a cancer patient may want, rather than a final decision to be treatment enough information about the reason for choosing a therapy by a 
doctor (Omid, 2011). Another study has been shown that the effective communication between patient and doctor prepares up to $80 \%$ of the patients to take the quick decision on their treatment as oncologist proposed (Gaberson, 2010). Disease on the patient and in their relatives usually exerts various stress such as fear, anxiety and uncertainty and is also full of cognitive errors. Mind at each stage of the reaction of the disease, prone to experience their own thoughts that come to mind way and may not reflect the realities of life. This type of thoughts is evaluated cognitive-behavioral therapy carefully, as known in negative automatic thoughts. The severity of such kinds of thoughts in individuals who have a psychiatric disease and improving treatment becomes more serious. As a result, patients seek delicate allusions to what they feel or think in their relationship with the medical staff. This information through verbal and/or nonverbal communication between patient and physician perhaps disclosed. It is therefore very important to establish effective communication between patient and physician, as well as thorough knowledge of specialists from automatic thoughts and psychological characteristics of patients, are essential. One of psychological intervention method that is effective in cancer patients is cognitive-behavioural therapy, which is used to identify and modify dysfunctional thoughts (automatic), relaxation techniques and problem-solving skills in reducing psychological distress such as anxiety, depression and physical problems like pain and fatigue (Gielissen et al., 2006. And the behavioural techniques such as relaxation, desensitization, and distraction for oncology different situations like anxiety associated with surgery process, are effective forecast to reducing or preventing symptoms of the condition such as nausea and vomiting associated with chemotherapy (Antoni et al., 2009). Cognitive model based on three "levels" of knowledge emphasizes: schema or core beliefs; assumptions or beliefs intermediate and automatic thoughts or ideas. The importance of such biases information processing and memory are considered. A general and absolute core beliefs are discussed such as (I am powerless, they are sympathetic to us, do always fatal cancer?). Intermediate beliefs (assumptions) and the rules of living in most situations and can be considered for conditional and/or unconditional statements. The following sentence is an example of conditional beliefs (my disease will go away if I practice my doctor's orders). Unconditioned intermediate beliefs are the following (my doctor's instructions should doalways). Depending on the position they are easier to identify automatic thoughts and core beliefs or beliefs, mediator. Each of cognitive levels was significantly linked with other levels. Nuclear beliefs, assumptions, and automatic thoughts are linked in terms of subject. The aim of this study is related to the scientific training in the treatment of cognitive-behavioural mental health of cancer patients, the residents of radiotherapy oncology in a small group, carried out this method of teaching assistants to oncology to provide effective communication between patient and physicians in identifying cognitive errors.

\section{Methodology:}

We adopted a quasi- experimental method in which data were collected before and after the intervention, where the participating are the residents attending the training program. The three types of residents in this study were selected, samples available in a small group. The research was carried out in Shohada Hospital and the Department of Radiotherapy - Oncology where two sessions of 2.5 hours in the form of workshops were conducted and proper filmization was done of it. In the first two sessions of radiation oncology, the respected field assistant test was taken. In the initial meeting with the patients, assistants, radiation oncologists and psychologists, the program was implemented in which the suffering caused due to their diagnosed disease, relevant treatment and related provided professionals' solutions to solve problems for patients were considered. During the meeting, as a part of practical, psychological aides familiar with how to solve common problems were explained. In view of the importance and requirements to be investigated further, the second session for the patients, with psychologists and specialists in oncology was arranged. Each session of the program included lectures, question and answer, the problem and the required solutions to resolve them were discussed at the end of the second meeting of Assistants test. Proper filmization was done of every test. Implementation of practical training was getting possible due to less number of assistants as well as patients.

In our study, the questionnaire included 12 items was our data collection tool. To search for papers in this field , seven domains cognitive errors involving catastrophizing (8 questions), mind reader (6 questions), all or nothing thinking (5 questions), underestimate the positive side (4 questions), should be (9 questions), extreme generalization (7 questions), bode (12 questions), a negative filter (10 questions), all or no thinking (11 questions). The first question raises questions about the effectiveness of the treatment patients are thinking.

\section{Results:}

In terms of demographic data, all the participants were married females within the age range of $30-35$ years. As per statistical analysis, the average groups before and after treatment are in Table 1 , the pre-test and post-test scores in the group studied considerably statistically significant ( $\mathrm{p}$ $<0.001$ ).

Regarding the pre-test and post-test scores, the statistical results revealed considerably significant. This 
reflects the success of training which shown by all the participants in the training sessions, the increased awareness of cognitive errors.

Table 1: Comparison of Average

\begin{tabular}{ll}
\hline Studied indicators & average \\
\hline Pre-test & $5 \cdot 37 \pm 1.42$ \\
Post-test & $9.2 \pm 1.29$ \\
difference & $3 \cdot 97 \pm 1.23$ \\
"p" value & $<0.001$ \\
\hline
\end{tabular}

Pre-test and post-test scores revealed the statistically signif icant difference. This reflects the success of training and shows all participants in the training sessions, the increased awareness of cognitive errors.

\section{Discussion \& conclusions:}

The present study, a repetitive type of research on science education in the treatment of cognitive-behavioural therapy on mental health of cancer patients, the residents of radiotherapy - oncology in a small group, with the aim of science teaching assistant. The effort to conduct this study was for the first time in the country, Iran.

In the above-conducted meetings, residents Radiation - Oncology focused on the methods of identifying patients' expectations with regard to their physical problems. How to deal and interact with the complaints and demands of patients in the relaxed atmosphere outside of the clinic and in the degree of congestion for other clients' practical were taught. We found that in such training sessions, Residents motive in mind to created recognizing the importance of their role in the elimination or reduction of psychological problems from patients and stress management in their precise continuation of such meetings can supplement their therapeutic activities and skills, to patients. Following feedback from residents, revealed the implementation of such programs and the continuation of pre-test and posttest results were evident, in particular, the usefulness of the program.

If the ultimate goal of the medical care is to provide optimal health outcomes for the patients, then an effective physician-patient relationship should also result in response to the patients' better health. Patient satisfaction, compliance and other results didn't correlate by any means with the patients' health status. Perhaps the results related to the trust and effective communication between patient and doctor showed some positive indications i.e., when a doctor is familiar with your thoughts and inefficient. If the patients get engaged in their cognitive errors, resulting in anxiety and negative emotions the patient to understand, communicate more effectively on the one hand and confidence with a doctor, on the other hand, makes the treatment faster than before (Benson et al., 2005). Kaplan et al., (1996) studied shown that the psychological aspects of emotional and more effective communication between patients and doctors cause the high level of the health improving in patients. The doctor-patient relationship must be an initial document that can act as a form of social support. Upto far extent our results also support the same hypothesis. The course has been able to improve the level of knowledge about the patient becomes psychological assistants.

\section{Acknowledgements:}

The authors would like to thank all participants for their kind collaboration.

\section{References:}

Antoni, M.H., Lechner, S., Diaz, A., Vargas, S., Holley, H., Phillips, K., McGregor, B., Carver, C.S. \& Blomberg, B. (2009): Cognitive behavioral stress management effects on psychosocial and physiological adaptation in women undergoing treatment for breast cancer. Brain Behav. Immun. 23(5):580-591.

Benson, J., Quince, T., Hibble, A., Fanshowe, T. \& Emery, J. (2005): Impact on patients of expanded, general practice based, student teaching: observational and qualitative study. BMJ., 331(7508):89.

Gaberson, K.B., Oermann, M.H. \& Shellenbarger, T. (2010): Clinical teaching strategies in nursing. 4 th ed. New York: Springer Publisher Company; pp. 456.

Gielissen, M.F., Verhagen, S., Witjes, F., Bleijenberg, G. (2006): Effects of cognitive behavior therapy in severely fatigued disease-free cancer patients compared with patients waiting for cognitive behavior therapy: a randomized controlled trial. J. Clin. Oncol., 24(30):4882-4887.

Kaplan, S.H., Greenf ield, S., Gandek, B., Rogers, W.H. \& Ware, J.E. (1996): Characteristics of physicians with participatory decision-making styles. Ann. Intern. Med. 124:497-504.

Lowe, M., Kerridge, I., McPhee, J., Hart, C. (2008): Do patients have an obligation to participate in student teaching? $\underline{\text { Med. }}$ Educ., 42(3):237-241.

Omid, A. (2011): A Qualitative Study of Faculty Members' Lived Experiences in Ward Round Teaching In Isfahan University of Medical Sciences. [Dissertation]. Tehran: Tehran medical school [in Persian].

Omrani , S., Fardanesh, H., Ebrahimzade, I., Sarmadi, M.R. \& Rezaei, M. (2013): Comparing the Effects of Lecture-Based and E-Learning Methods on Learning and Motivation of Participants in Continuing Medical Education. Strides Dev. Med. Educ., 9(2):143-152.

Soroush, M., Hejazi, E., Shoakazem, S.M. \& Gheranpayeh, L. (2015): Body Image Psychological Characteristics and Hope in Women with Breast Cancer. Iranian Qua. J. Breast Dis., $7(4): 52-63$

Spencer, J. (2003): ABC of learning and teaching in medicne: learning and teaching in the clinical environment. $B M J$; 326:591.

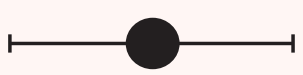

